\title{
The $10^{5} L_{\odot}$ high-mass protostellar object IRAS $23151+5912$
}

\author{
H. Beuther ${ }^{1}$, Q. Zhang ${ }^{2}$, T. R. Hunter ${ }^{3}$, T. K. Sridharan ${ }^{2}$, and E. A. Bergin ${ }^{4}$ \\ 1 Max-Planck-Institute for Astronomy, Königstuhl 17, 69117 Heidelberg, Germany \\ e-mail: beuther@mpia.de \\ 2 Harvard-Smithsonian Center for Astrophysics, 60 Garden Street, Cambridge, MA 02138, USA \\ e-mail: name@cfa.harvard.edu \\ 3 NRAO, 520 Edgemont Rd, Charlottesville, VA 22903, USA \\ e-mail: thunter@nrao.edu \\ ${ }^{4}$ University of Michigan, 825 Dennison Building, 500 Church Street, Ann Arbor, MI 48109-1042, USA \\ e-mail: ebergin@umich.edu
}

Received 1 June 2007 / Accepted 18 July 2007

\section{ABSTRACT}

\begin{abstract}
Context. While most sources above $10^{5} L_{\odot}$ have already formed an Ultracompact HII region (UCHII), this is not necessarily the case for sources of lower luminosity. Characterizing sources in the transition phase, i.e., very luminous objects without any detectable free-free emission, is important for a general understanding of massive star formation.

Aims. We Characterize one of the most luminous High-Mass Protostellar Objects (HMPO) that has not yet formed any detectable UCHII region.

Methods. The region was observed with the Submillimeter Array in three different array configurations at $875 \mu \mathrm{m}$ in the submm continuum and spectral line emission at sub-arcsecond resolution.

Results. The $875 \mu \mathrm{m}$ submm continuum emission has been resolved into at least two condensations. The previously believed driving source of one of the outflows, the infrared source IRS1, is $\sim 0.9^{\prime \prime}$ offset from the main submm peak. The data do not allow to differentiate whether this offset is real, either caused by different sources or a shift of the photo-center due to scattering, or whether it is only due to poor astrometry of the infrared data. Over the entire $4 \mathrm{GHz}$ bandwidth we detect an intermediate dense spectral line forest with 27 lines from 8 different species, isotopologues or vibrationally-torsionally excited states. Temperature estimates based on the $\mathrm{CH}_{3} \mathrm{OH}$ line series result in values of $T$ (Peak1) $\sim 150 \pm 50 \mathrm{~K}$ and $T$ (Peak2) $\sim 80 \pm 30 \mathrm{~K}$ for the two submm peak positions, respectively. The $\mathrm{SiO}(8-7)$ red- and blue-shifted line maps indicate the presence of two molecular outflows. In contrast, the vibrationally-torsionally excited $\mathrm{CH}_{3} \mathrm{OH}$ line exhibits a velocity gradient approximately perpendicular to one of the outflows. With a size of approximately $5000 \mathrm{AU}$ and no Keplerian rotation signature, this structure does not resemble a genuine accretion disk but rather a larger-scale rotating toroid that may harbor a more common accretion disk at its so far unresolved center.
\end{abstract}

Key words. stars: formation - stars: early-type - stars: individual: IRAS 23151+5912 - ISM: dust, extinction - ISM: jets and outflows

\section{Introduction}

In contrast to known Ultracompact HII regions (UCHIIs) with luminosities sometimes even exceeding $10^{6} L_{\odot}$ (e.g., Wood \& Churchwell 1989), a close inspection of large samples of younger High-Mass Protostellar Objects (HMPOs) (e.g., Molinari et al. 1996; Sridharan et al. 2002; Faúndez et al. 2004; Beltrán et al. 2006) shows that only very few sources at that evolutionary stage exceed $10^{5} L_{\odot}$. The average luminosity difference between HMPOs and UCHIIs is likely attributed to evolutionary differences, i.e., the still accreting HMPO will gain more mass eventually being energetic enough to produce detectable free-free emission at $\mathrm{cm}$ wavelengths and hence evolve into an UCHII region (e.g., Sridharan et al. 2002; Beuther et al. 2007a). Observations indicate that below $10^{5} L_{\odot}$ the free-free emission of the forming star is still potentially quenched, either by the pressure of the infalling gas, high recombination rates due to high accretion and infall rates, or by gravitational trapping (e.g., Walmsley 1995; Keto 2003). However, since almost all massive star-forming regions above $10^{5} L_{\odot}$ are detected at $\mathrm{cm}$ wavelengths, these processes appear less feasible in that regime. Most interferometric investigations focused either on very luminous UCHII regions (e.g., Cesaroni et al. 1994; Keto 2002;
Beltrán et al. 2004; Hoare et al. 2007) or on younger but less luminous HMPOs (e.g., Shepherd et al. 1998; Zhang et al. 1998; Cesaroni et al. 1999; Beuther et al. 2002a, 2005c). This transition phase of a source that has already a very luminous and massive accreting protostar, however, that is yet not capable to ionize enough gas to be detected as a Hypercompact HII region (HCII), has not been studied well so far. Therefore, here we present new Submillimeter Array $\left(\mathrm{SMA}^{1}\right)$ data observed toward the $10^{5} L_{\odot}$ HMPO IRAS 23151+5912.

The rationale to choose IRAS $23151+5912$ was foremost based on two criteria: the source had to be very luminous $\left(\geq 10^{5} L_{\odot}\right.$ ) and it had to be very young, in an evolutionary stage prior to forming any detectable ultracompact HII region. The source IRAS 23151+5912 comprised both features, hence it is one of the most luminous HMPOs in a pre-UCHII region phase. The source at a kinematic distance of $5.7 \mathrm{kpc}$ is part of a large sample of 69 candidate HMPOs initially studied by Sridharan et al. (2002); Beuther et al. (2002b,c,d). IRAS 23151+5912

\footnotetext{
${ }^{1}$ The Submillimeter Array is a joint project between the Smithsonian Astrophysical Observatory and the Academia Sinica Institute of Astronomy and Astrophysics, and is funded by the Smithsonian Institution and the Academia Sinica.
} 
shows $\mathrm{H}_{2} \mathrm{O}$ maser emission and it is not detected in the $\mathrm{cm}$ band at $8 \mathrm{GHz}$ down to $1 \mathrm{mJy}$ (Tofani et al. 1995; Sridharan et al. 2002), thus no significant hypercompact HII region has formed yet (Sridharan et al. 2002). Based on single-dish $1.2 \mathrm{~mm}$ dust continuum observations the total gas mass within the region is estimated to $\sim 600 M_{\odot}$ (Beuther et al. 2002b, 2005a). Observations of the $\mathrm{CO}(2-1)$ and $\mathrm{SiO}(2-1)$ lines with the IRAM 30 m telescope reveal a bipolar outflow with an entrained gas mass of $\sim 20 M_{\odot}$ (Beuther et al. 2002c). The derived outflow rate of $1 \times 10^{-3} M_{\odot} \mathrm{yr}^{-1}$ translates into an estimate of the accretion rate of the same order of magnitude (Beuther et al. 2002c). Recent interferometric high-spatial-resolution $\mathrm{SiO}(2-1)$ observations reveal a quasi-parabolic blue-shifted outflow cone being consistent with a wide-angle wind (Qiu et al. 2007). Weigelt et al. (2006) speckle-imaged the region in the near-infrared $\mathrm{K}$-band, and the nebulosity they find coincides with the blue outflow wing. Their data rather suggest that the outflow features are produced by a precessing jet. In addition, Weigelt et al. (2006) find an embedded K-band source IRS1 which they propose to be the driving source of the molecular outflow. Furthermore, $\mathrm{NH}_{3}$ inversion lines from the $(1,1)$ to the $(4,4)$ transitions have been detected with the Effelsberg $100 \mathrm{~m}$ telescope (unpublished data, Beuther priv. comm.), the excitation temperature of the $(4,4)$ line is about $200 \mathrm{~K}$. The $\mathrm{NH}_{3}(1,1) /(4,4)$ line ratio of only $\sim 2$ indicates warm and dense gas at the core center. Very recently, Garay et al. (2007) detected an unresolved hypercompact HII region at a sub-mJy flux level toward IRAS $23151+5912$. Furthermore, they detected about $30^{\prime \prime}$ apart a more evolved cometary HII region. While the latter may contribute part of the total luminoisty of the region ( $20 \%$, Garay et al. 2007), the main power-house of the region very likely remains the HMPO IRAS 23151+5912. Therefore, our target source comprises all features of a luminous High-Mass Protostellar Object at an early evolutionary stage. Assuming that the source is still in its accretion phase, it has not yet reached its final luminosity (e.g., Sridharan et al. 2002), and the total luminosity of the forming cluster will even exceed $10^{5} L_{\odot}$ during its ongoing evolution.

\section{Observations}

We have observed the HMPO IRAS 23151+5912 with the Submillimeter Array during three nights between May and November 2005. We used three different array configurations (compact, extended, very extended) with unprojected baselines between 16 and $500 \mathrm{~m}$, resulting at $875 \mu \mathrm{m}$ in a projected baseline range from 8 to $591 \mathrm{k} \lambda$. The chosen phase center was RA [J2000.0]: $23^{\mathrm{h}} 17^{\mathrm{m}} 21^{\mathrm{s}} .0$ and Dec [J2000.0] +59 $28^{\prime} 48^{\prime \prime} .995$. The velocity of rest is $v_{\text {lsr }} \sim-54.4 \mathrm{~km} \mathrm{~s}^{-1}$.

For bandpass calibration we used 3C 279 and 3C 454.3. The flux scale was derived in the very extended configuration from observations of $3 \mathrm{C} 279$. For the extended and compact configurations, we used $3 \mathrm{C} 454.3$ for the relative scaling between the various baselines and then scaled that absolutely via observations of Uranus. The flux accuracy is estimated to be accurate within $20 \%$. Phase and amplitude calibration was done via frequent observations of the quasars J0102+584 and BL Lac, about $13.5^{\circ}$ and $20.7^{\circ}$ from the phase center. The zenith opacity $\tau(348 \mathrm{GHz})$, estimated from the measurements of the NRAO tipping radiometer located at the Caltech Submillimeter Observatory, varied between the different observations nights between $\sim 0.1$ and $\sim 0.3$ (scaled from the $225 \mathrm{GHz}$ measurement, $\tau(348 \mathrm{GHZ}) \sim 2.5 \times \tau(225 \mathrm{GHz}))$. The receiver operated in a double-sideband mode with an IF band of $4-6 \mathrm{GHz}$ so that the upper and lower sideband were separated by $10 \mathrm{GHz}$. The
Table 1. Peak fluxes densities, rms and synthesized beams of integrated line images in Fig. 3.

\begin{tabular}{lrrr}
\hline \hline Line & $\begin{array}{r}S_{\text {peak }} \\
\frac{\text { mJy }}{\text { beam }}\end{array}$ & $\begin{array}{r}\text { rms } \\
\frac{\text { JJy }}{\text { beam }}\end{array}$ & $\begin{array}{r}\text { Beam } \\
\text { "(PA) }\end{array}$ \\
\hline $\mathrm{CH}_{3} \mathrm{OH}\left(7_{3,5}-6_{2,4}\right)$ & 1014 & 79 & $0.8 \times 0.67(19)$ \\
$\mathrm{CH}_{3} \mathrm{OH}\left(7_{4,3}-6_{4,3}\right), v_{\mathrm{t}}=1$ & 617 & 80 & $1.0 \times 0.81(19)$ \\
$\mathrm{C}^{34} \mathrm{~S}(7-6)$ & 411 & 64 & $1.08 \times 0.87(18)$ \\
$\mathrm{SiO}(8-7)$ & 433 & 59 & $1.14 \times 0.89(80)$ \\
$\mathrm{SO}_{2}\left(24_{2,22}-23_{3,21}\right)$ & 826 & 60 & $1.07 \times 0.84(80)$ \\
${ }^{34} \mathrm{SO}\left(8_{8}-7_{7}\right)$ & 977 & 67 & $1.08 \times 0.87(19)$ \\
\hline
\end{tabular}

central frequencies of the upper and lower sideband were 348.2 and $338.2 \mathrm{GHz}$, respectively. The correlator had a bandwidth of $2 \mathrm{GHz}$ and the channel spacing was $0.8125 \mathrm{MHz}$. Measured double-sideband system temperatures corrected to the top of the atmosphere were between 100 and $1000 \mathrm{~K}$, depending on the zenith opacity and the elevation of the source. Our sensitivity was dynamic-range limited by the side-lobes of the strongest emission peaks and thus varied between the line maps of different molecules and molecular transitions. This limitation was due to the incomplete sampling of short uv-spacings and the presence of extended structures. The $1 \sigma \mathrm{rms}$ for the velocity-integrated molecular line maps (the velocity ranges for the integrations were chosen for each line separately depending on the line-widths and intensities) ranged between 59 and $80 \mathrm{mJy} \mathrm{beam}^{-1}$ (Table 1). The average synthesized beam of the spectral line maps was $1^{\prime \prime} \times 0.8^{\prime \prime}$ (Table 1 ). The $875 \mu \mathrm{m}$ submm continuum image was created by averaging the line-free parts of both sidebands. The $1 \sigma \mathrm{rms}$ of the submm continuum image was $\sim 4.9 \mathrm{mJy} / \mathrm{beam}$, and the achieved synthesized beam was $0.57^{\prime \prime} \times 0.48^{\prime \prime}\left(\mathrm{PA} 86^{\circ}\right)$. The initial flagging and calibration was done with the IDL superset MIR originally developed for the Owens Valley Radio Observatory (Scoville et al. 1993) and adapted for the SMA ${ }^{2}$. The imaging and data analysis were conducted in MIRIAD (Sault et al. 1995).

\section{Results}

\section{1. $875 \mu m$ continuum emission in IRAS $23151+5912$}

Figure 1 shows the $875 \mu \mathrm{m}$ continuum image extracted from the line-free part of the data-cube. The spatial resolution of $0.57^{\prime \prime} \times 0.48^{\prime \prime}$ corresponds to $\sim 3000 \mathrm{AU}$ at the given distance of $\sim 5.7 \mathrm{kpc}$. The peak and integrated fluxes of the main peak 1 are about double of those from the minor peak 2 (Table 2). However, one of the $\mathrm{H}_{2} \mathrm{O}$ maser positions is clearly associated with submm peak 2 and none with submm peak 1 . The second $\mathrm{H}_{2} \mathrm{O}$ maser position is right between both submm peak positions. Interestingly, the infrared source IRS1 detected by Weigelt et al. (2006) is offset by about $0.9^{\prime \prime}$ north-east of the main submm peak position. The positional accuracy of the interferometric submm data is estimated to be $\sim 0.1^{\prime \prime}$. Contrary to that, the astrometry of the infrared data is based on the 2MASS astrometry, and this is estimated in this field to be not better than $1^{\prime \prime}$ (Preibisch, priv. comm.). Therefore, we cannot unambiguously decide whether IRS1 and the submm peak 1 are the same or different sources. Based on the estimated $\mathrm{H}_{2}$ column densities of the order $10^{24} \mathrm{~cm}^{-2}$ (corresponding to visual extinctions of the order 1000, see below), one would not expect to detect nearinfrared emission from the submm peaks. However, arguments

\footnotetext{
2 The MIR cookbook by Charlie Qi can be found at http://cfa-www . harvard. edu/ cqi/mircook . html
} 
Table 2. Submm continuum source parameters.

\begin{tabular}{lrrrrrr}
\hline \hline Source & $\begin{array}{r}\text { RA } \\
{[\mathrm{J} 2000]}\end{array}$ & $\begin{array}{r}\text { Dec } \\
{[\mathrm{J} 2000]}\end{array}$ & $\begin{array}{r}S_{\text {peak }} \\
{\left[\frac{\mathrm{mJy}}{\text { beam }}\right]}\end{array}$ & $\begin{array}{r}S_{\text {int }} \\
{[\mathrm{mJy}]}\end{array}$ & $\begin{array}{r}M \\
{\left[M_{\odot}\right]}\end{array}$ & $\begin{array}{r}N \\
{\left[10^{24} \mathrm{~cm}^{-3}\right]}\end{array}$ \\
\hline Peak 1 & $23: 17: 20.916$ & $59: 28: 47.65$ & 81 & 282 & 26 & 2.3 \\
Peak 2 & $23: 17: 20.800$ & $59: 28: 47.17$ & 44 & 129 & 12 & 1.2 \\
\hline
\end{tabular}

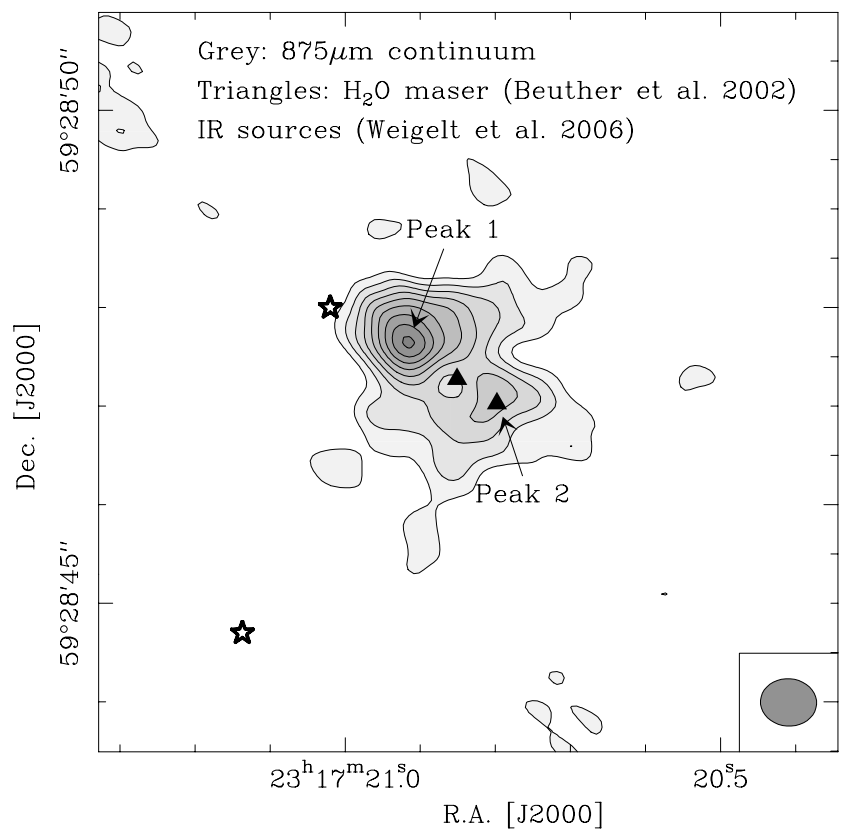

Fig. 1. The grey-scale with contours shows the $875 \mu \mathrm{m}$ continuum emission. The contours go from $\pm 18(3 \sigma)$ to $\pm 98 \%$ (step $\pm 10 \%$ ) from the peak flux of $80.8 \mathrm{mJy} / \mathrm{beam}$. The two main submm peaks are marked and the triangles and stars show the positions of $\mathrm{H}_{2} \mathrm{O}$ maser and nearinfrared peaks from Beuther et al. (2002d) and Weigelt et al. (2006). The synthesized beam of $0.57^{\prime \prime} \times 0.48^{\prime \prime}$ is shown at the bottom right.

based on the outflow emission may explain this (see Sect. 4.2). Furthermore, Qiu et al. (2007) detected more extended 3.4 and $1.3 \mathrm{~mm}$ emission west of the peak. Although their $1.3 \mathrm{~mm}$ feature is only at a $2-3 \sigma$ level, the morphological similarity with the $3.4 \mathrm{~mm}$ data and the infrared/SiO outflow stimulates the speculation that this $\mathrm{mm}$ emission may be caused by the outflow. Extrapolating the $1.3 \mathrm{~mm}$ flux density of $\sim 4.25 \mathrm{mJy}$ with a typical jet-frequency dependence of $v^{0.6}$ to our continuum frequency of $875 \mu \mathrm{m}$, the expected submm flux density would be $S(875 \mu \mathrm{m}) \sim 5.4 \mathrm{mJy}$, only barely above our $1 \sigma$ noise level. Therefore, it is no surprise that we cannot detect these features in the submm data.

Table 2 lists the absolute source positions, their $875 \mu \mathrm{m}$ peak fluxes and the integrated flux density approximately associated with the two sub-sources. Assuming that the observed submm continuum emission is due to optically thin dust emission, we can calculate gas masses and gas column densities following Hildebrand (1983) and Beuther et al. (2002b, 2005a). Since IRAS23151+5912 is in a very early evolutionary stage we use the dust temperature derived from the IRAS data $(68 \mathrm{~K}$, Sridharan et al. 2002) and a dust opacity index $\beta=2$, corresponding to a dust opacity per unit mass of $\kappa(875 \mu \mathrm{m}) \sim$ $0.8 \mathrm{~cm}^{2} \mathrm{~g}^{-1}$. Given the uncertainties in $\beta$ and $T$, we estimate the masses to be correct within a factor $2-5$. Table 2 gives the derived masses and column densities separated for each of the two peaks. While the two core masses vary between 26 and $12 M_{\odot}$, the column densities are of the order $10^{24} \mathrm{~cm}^{-2}$, corresponding

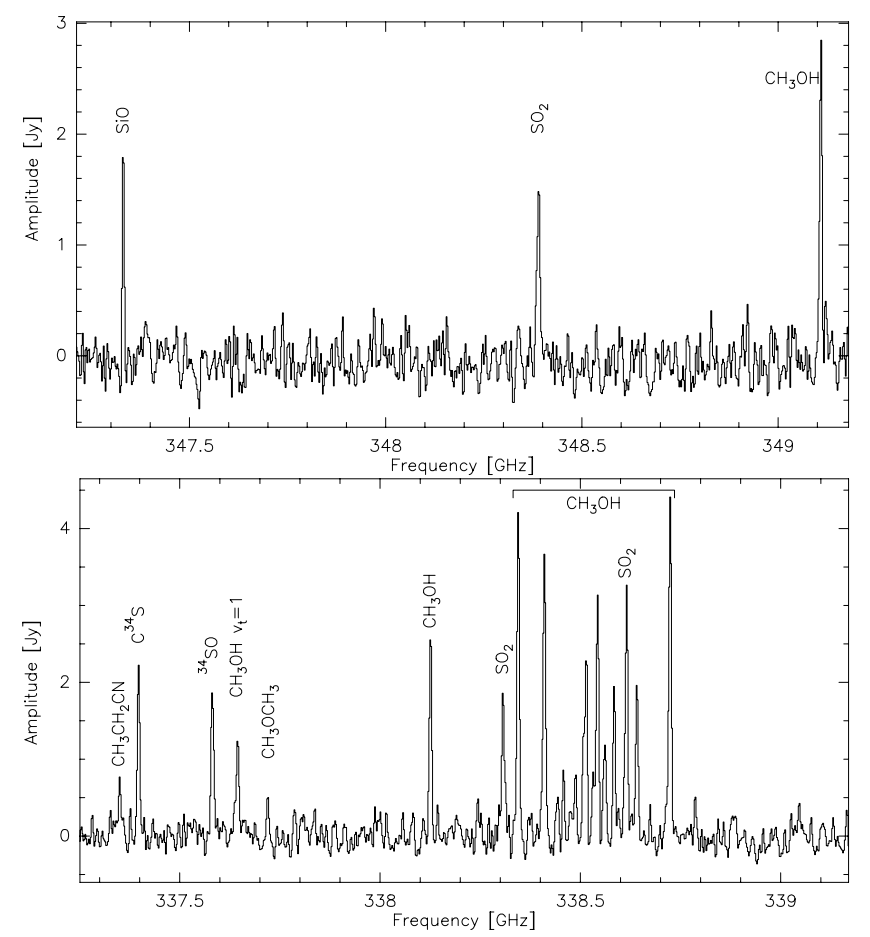

Fig. 2. Upper and lower sideband (top and bottom panel) spectra taken in the uv-domain on a short baseline of $\sim 14 \mathrm{~m}$. The chosen spectral resolution is $2 \mathrm{~km} \mathrm{~s}^{-1}$.

to extremely high visual extinctions of the order $1000\left(A_{\mathrm{v}}=\right.$ $N_{\mathrm{H}} / 0.94 \times 10^{21}$, Frerking et al. 1982). Integrating the whole submm continuum emission shown in Fig. 1 ( $\left.S_{\text {int }} \sim 404 \mathrm{mJy}\right)$, the total detected mass is $\sim 38 M_{\odot}$. Comparing the integrated flux density with the peak flux observed with the SCUBA array on the JCMT $\left(\sim 15^{\prime \prime}\right.$ resolution) at $850 \mu \mathrm{m}$ of $1890 \mathrm{mJy}$ (Williams et al. 2004), approximately $80 \%$ of the total flux is filtered out in our interferometer observations ${ }^{3}$. The $3 \sigma$ rms of the continuum image of $\sim 14.7 \mathrm{mJy}$ corresponds to a mass sensitivity of $\sim 1.4 M_{\odot}$ at $68 \mathrm{~K}$. Assuming lower temperatures offset from the peak, the $3 \sigma$ value corresponds to a higher mass sensitivity, e.g., $\sim 6.4 M_{\odot}$ at $20 \mathrm{~K}$.

\subsection{Submm spectral lines in IRAS $23151+5912$}

The spectral bandwidths of $4 \mathrm{GHz}$ in total revealed 27 spectral lines (Fig. 2 and Table 3). We detected a series of groundstate and vibrationally-torsionally excited $\mathrm{CH}_{3} \mathrm{OH}$ lines, some sulphur-bearing species $\left({ }^{34} \mathrm{SO}, \mathrm{SO}_{2}, \mathrm{C}^{34} \mathrm{~S}\right)$, the outflow- and shock-tracing $\mathrm{SiO}$ and a few more complex molecules like $\mathrm{CH}_{3} \mathrm{CH}_{2} \mathrm{CN}$ and $\mathrm{CH}_{3} \mathrm{OCH}_{3}$. The excitation temperatures of especially the $\mathrm{CH}_{3} \mathrm{OH}$ lines cover a broad range from approximately 40 to $360 \mathrm{~K}$ (Table 3 ).

\footnotetext{
3 Using only the compact configuration data, still approximately $60 \%$ of the single-dish emission remain filtered out.
} 
Table 3. Line parameters.

\begin{tabular}{|c|c|c|c|}
\hline $\begin{array}{l}\text { Freq. } \\
\mathrm{GHz}\end{array}$ & Line & $\begin{array}{r}E_{u} \\
\mathrm{~K}\end{array}$ & $\begin{array}{r}1 \sigma \\
{\left[\frac{\mathrm{mJy}}{\text { beam }}\right]}\end{array}$ \\
\hline 337.348 & $\mathrm{CH}_{3} \mathrm{CH}_{2} \mathrm{CN}\left(38_{3,36}-37_{3,35}\right)$ & 328 & \\
\hline 337.397 & $C^{34} S(7-6)$ & 65 & 60 \\
\hline 337.582 & ${ }^{34} \mathrm{SO}\left(8_{8}-7_{7}\right)$ & 86 & 70 \\
\hline 337.642 & $\mathrm{CH}_{3} \mathrm{OH}\left(7_{1,7}-6_{1,6}\right) E\left(v_{\mathrm{t}}=1\right)$ & 356 & 80 \\
\hline 337.722 & $\mathrm{CH}_{3} \mathrm{OCH}_{3}\left(7_{4,4}-6_{3,3}\right) \mathrm{EE}$ & 48 & \\
\hline 338.125 & $\mathrm{CH}_{3} \mathrm{OH}\left(7_{0,7}-6_{0,6}\right) \mathrm{E}$ & 78 & \\
\hline 338.306 & $\mathrm{SO}_{2}\left(14_{4,14}-18_{3,15}\right)$ & 197 & \\
\hline 338.345 & $\mathrm{CH}_{3} \mathrm{OH}\left(7_{1,7}-6_{1,6}\right) \mathrm{E}$ & 71 & \\
\hline 338.409 & $\mathrm{CH}_{3} \mathrm{OH}\left(7_{0,7}-6_{0,6}\right) \mathrm{A}$ & 65 & \\
\hline 338.457 & $\mathrm{CH}_{3} \mathrm{OH}\left(7_{5,2}-6_{5,1}\right) \mathrm{E}$ & 189 & \\
\hline \multirow[t]{2}{*}{338.486} & $\mathrm{CH}_{3} \mathrm{OH}\left(7_{5,3}-6_{5,2}\right) \mathrm{A}$ & 203 & \\
\hline & $\mathrm{CH}_{3} \mathrm{OH}\left(7_{5,2}-6_{5,1}\right) \mathrm{A}^{-}$ & 203 & \\
\hline \multirow[t]{3}{*}{338.513} & $\mathrm{CH}_{3} \mathrm{OH}\left(7_{4,4}-6_{4,3}\right) \mathrm{A}^{-}$ & 145 & \\
\hline & $\mathrm{CH}_{3} \mathrm{OH}\left(7_{4,3}-6_{4,2}\right) \mathrm{A}$ & 145 & \\
\hline & $\mathrm{CH}_{3} \mathrm{OH}\left(7_{2,6}-6_{2,5}\right) \mathrm{A}^{-}$ & 103 & \\
\hline 338.530 & $\mathrm{CH}_{3} \mathrm{OH}\left(7_{4,3}-6_{4,2}\right) \mathrm{E}$ & 161 & \\
\hline 338.541 & $\mathrm{CH}_{3} \mathrm{OH}\left(7_{3,5}-6_{3,4}\right) \mathrm{A}^{+}$ & 115 & \\
\hline 338.543 & $\mathrm{CH}_{3} \mathrm{OH}\left(7_{3,4}-6_{3,3}\right) \mathrm{A}^{-}$ & 115 & \\
\hline 338.560 & $\mathrm{CH}_{3} \mathrm{OH}\left(7_{3,5}-6_{3,4}\right) \mathrm{E}$ & 128 & \\
\hline 338.583 & $\mathrm{CH}_{3} \mathrm{OH}\left(7_{3,4}-6_{3,3}\right) \mathrm{E}$ & 113 & \\
\hline 338.612 & $\mathrm{SO}_{2}\left(20_{1,19}-19_{2,18}\right)$ & 199 & \\
\hline 338.640 & $\mathrm{CH}_{3} \mathrm{OH}\left(7_{2,5}-6_{2,4}\right) \mathrm{A}$ & 103 & \\
\hline 338.722 & $\mathrm{CH}_{3} \mathrm{OH}\left(7_{2,5}-6_{2,4}\right) \mathrm{E}$ & 87 & 80 \\
\hline 338.723 & $\mathrm{CH}_{3} \mathrm{OH}\left(7_{2,6}-6_{2,5}\right) \mathrm{E}$ & 91 & \\
\hline 347.331 & ${ }^{28} \mathrm{SiO}(8-7)$ & 75 & 60 \\
\hline 348.388 & $\mathrm{SO}_{2}\left(24_{2,22}-23_{3,21}\right)$ & 293 & 58 \\
\hline 349.107 & $\mathrm{CH}_{3} \mathrm{OH}\left(14_{1,13}-14_{0,14}\right)$ & 43 & \\
\hline
\end{tabular}

Excluding the two spectral lines from the more complex molecules $\mathrm{CH}_{3} \mathrm{CH}_{2} \mathrm{CN}$ and $\mathrm{CH}_{3} \mathrm{OCH}_{3}$ that were too weak for imaging, we were able to image the molecular emission of all other species (Fig. 3). The three spectral line images from $\mathrm{CH}_{3} \mathrm{OH}, \mathrm{SO}_{2}$ and ${ }^{34} \mathrm{SO}$ all peak in the near vicinity of the main submm peak and show emission approximately associated with the region of submm continuum emission. This is partly different for the three other line images. The integrated $\mathrm{SiO}(8-$ 7) emission shows a relatively complex morphology which is largely caused by the molecular outflows in this region (see Sect. 4.1). The virbrationally-torsionally excited $\mathrm{CH}_{3} \mathrm{OH}$ emission shows a double-peaked integrated map around the main submm peak 1 and may be attributed to rotation from a largerscale toroid around the most massive protostellar obect(s) (see Sect. 4.4). Furthermore, the $\mathrm{C}^{34} \mathrm{~S}$ emission is centered offset from the submm continuum peaks and shows relatively strong more extended emission reminiscent to the recent observations of the hot molecular core G29.96 (Beuther et al. 2007b).

Compared with hot cores like Orion-KL (Beuther et al. 2005b) or G29.96 (Beuther et al. 2007b) IRAS 23151+5912 exhibits relatively few spectral lines, however, it shows more lines than lower luminosity HMPOs like IRAS 05358+3543 (Leurini et al., submitted to A\&A). In a follow-up paper we present a more detailed comparison between all these sources with a special emphasis on the chemical diversity and a potential chemical evolutionary sequence (Beuther et al., submitted).

\section{Discussion}

\subsection{Multiple molecular outflows}

The $\mathrm{SiO}(8-7)$ emission allows us to investigate the molecular outflow(s) in this region. However, in comparison to the $\mathrm{SiO}(2-1)$ emission with a velocity spread from -70 to $-48 \mathrm{~km} \mathrm{~s}^{-1}$ (Qiu et al. 2007), the observable total velocity spread in the $J=8-7$ line is only from -60 to $-51 \mathrm{~km} \mathrm{~s}^{-1}$, relatively close to the velocity of the ambient gas of $-54.4 \mathrm{~km} \mathrm{~s}^{-1}$ (Sridharan et al. 2002). The difference between the $J=2-1$ and $J=8-7$ lines can likely be explained by the higher excitation temperature of the latter line. The energy levels above ground $E_{u} / k$ are 6 and $75 \mathrm{~K}$ for the lower and higher excited lines, respectively. With typical gas temperatures in molecular outflows of the order $30 \mathrm{~K}$ (e.g., Cabrit \& Bertout 1992), the $J=8-7$ line is much harder to excite and hence more difficult to detect at all. Separating spectral core from line wing emission is not an easy task when no pronounced broad line wings are observable, however, since $\mathrm{SiO}$ is believe to be produced almost exclusively by sputtering from dust grains (e.g., Schilke et al. 1997), it is likely that most of the $\mathrm{SiO}$ emission is caused by the molcular outflow. Therefore, one can produce outflow maps from $\mathrm{SiO}$ data going closer to the ambient gas velocities than it is feasible for more common molecules like CO. Hence, we imaged the blue- and red-shifted $\mathrm{SiO}(8-7)$ emission in the intervals $[-60,-56] \mathrm{km} \mathrm{s}^{-1}$ (blue) and $[-54,-51] \mathrm{km} \mathrm{s}^{-1}$ (red), respectively. Figure 4 presents this $\mathrm{SiO}$ line wing maps as overlay on the submm continuum emission. The blue- and red-shifted emission are spatially distinct, but the morphology is more ambiguous than would be expected from the previous $\mathrm{mm}$ and nearinfrared outflow observations (e.g., Beuther et al. 2002c; Weigelt et al. 2006; Qiu et al. 2007). These previous observations clearly identify one outflow in approximately east-west direction emanating from the region containing the main submm peak and the infrared source IRS1. This east-west outflow can be recognized in the new $\mathrm{SiO}(8-7)$ data as well, although one should note that the geometric center of the blue- and red-shifted peak is not exactly the main submm continuum peak but offset about $0.5^{\prime \prime}$ to the west. Furthermore, there is considerable additional $\mathrm{SiO}$ blue and red-shifted emission north and south of the continuum sources, respectively. This emission is offset from the dust continuum peaks and hence unlikley be caused by the ambient gas. Since we have at least two embedded protostellar sources, we suggest that we are witnessing two outflows emanating from the region as well. Reinspecting the $\mathrm{SiO}(2-1)$ observations from Qiu et al. (2007), one finds that their lower excitation data show an additional red-shifted isolated peak toward the south as well. Our SMA data were observed in three different configurations having a relatively good uv-sampling and hence spatial coverage, however, the southern peak from Qiu et al. (2007) is close to the edge of our primary beam and thus difficult to detect anyway. It is likely that the $\mathrm{SiO}$ observations by Qiu et al. (2007) filtered out more of the extended emission which could cause the relative isolation of the southern $\mathrm{SiO}$ peak in their data.

\subsection{The outflow driving sources}

Based on a faint jet-like infrared feature emanating from IRS1, Weigelt et al. (2006) propose that this should be the driving source of the east-west molecular outflow. In contrast to that, one would expect that powerful outflows like that in IRAS $23151+5912$ are driven by younger and more deeply embedded sources like the submm peak 1 . While we cannot astrometrically decide whether the two sources are the same or not, the outflow associations indicate that the infrared and submm emission may indeed emanate from the same massive protostellar object. Furthermore, due to scattering effects within embedded objects it is possible that the emission peak shifts at near-infrared wavelengths in the direction of the steepest density 


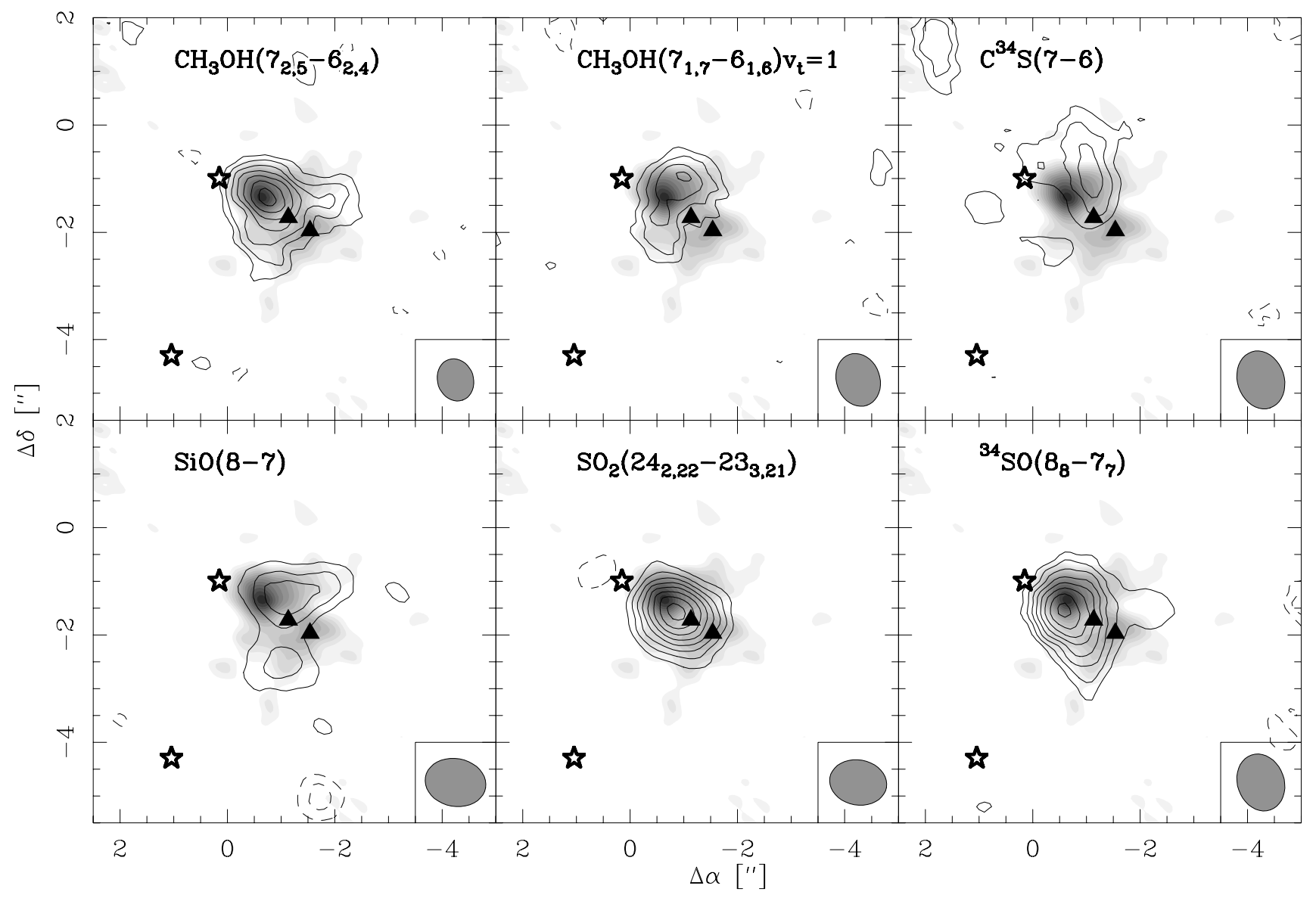

Fig. 3. Integrated images in IRAS $23151+5912$ of the molecules identified in Fig. 2 that are strong enough for imaging. The species are labeld in each panel and the synthesized beams are shown at the bottom left of each panel. The grey-scale shows the submm continuum emission as presented in Fig. 1. Triangles and stars mark the $\mathrm{H}_{2} \mathrm{O}$ maser and near-infrared peaks from Beuther et al. (2002d) and Weigelt et al. (2006). The contour levels start at $\pm 3 \sigma$ and continue in $\pm 1.5 \sigma$ steps, the $1 \sigma$ values are given in Table 3 .

gradient which is toward the outflow cavity (e.g., Eisner et al. 2005; Stark et al. 2006). Therefore, while an outflow cavity allows to penetrate deeper into the core than possible in spherical symmetry, the scattering effects could cause even a real offset between the infrared and submm source. Hence, although the nearinfared source IRS1 and the submm peak 1 may be two separate sources, the data are also consistent with both emission peaks potentially being due to the same embedded young and massive protostellar source that could drive the east-west outflow.

The association of one of the maser peaks with submm peak 2 potentially indicates that this source could be driving a molecular outflow as well. To check this hypothesis, we produced a position-velocity diagram through submm peak 2 at a position angle of 160 degrees from north, proximately the orientation of the 2nd proposed outflow (Fig. 5). While it is less clear for the blue-shifted emission, the red-shifted side shows a clear increase of velocity with distance from submm peak 2 , resembling the Hubble-law for molecular outflows (e.g., Richer et al. 2000). Based on the morphology and the position-velocity structure of the north-east south-west outflow, we suggest that this outflow is driven by the embedded source within submm peak 2.

The second $\mathrm{H}_{2} \mathrm{O}$ maser position right between the two submm peaks may either be caused solely by shock interactions of a molecular outflow with the ambient gas, or it could be associated with a low-mass source which is below our detection threshhold ( $3 \sigma$ continuum sensitivity corresponds to $\sim 1.4 M_{\odot}$ at $68 \mathrm{~K}$ or a higher value of $\sim 6.4 M_{\odot}$ at lower temperatures of $20 \mathrm{~K}$, Sect. 3.1) and hence not possible to be identified as an additional weak submm peak. Although $\mathrm{H}_{2} \mathrm{O}$ masers are more often found toward massive star-forming regions (e.g., Forster \& Caswell 1999), there exist several examples of $\mathrm{H}_{2} \mathrm{O}$ maser emission from low-mass regions (Wilking et al. 1994; Claussen et al. 1998) as well as from lower-mass companions in regions of high-mass star formation (Hunter et al. 1999).

\subsection{The $\mathrm{CH}_{3} \mathrm{OH}$ line forest}

The detection of $19 \mathrm{CH}_{3} \mathrm{OH}$ lines with excitation temperatures between 43 and $356 \mathrm{~K}$ allows the temperature to be estimated toward the two submm continuum peaks. We modeled the whole lower sideband $\mathrm{CH}_{3} \mathrm{OH}$ spectrum toward both position in the local thermodynamic equilibrium approximation using the XCLASS superset to the CLASS software developed by Peter Schilke (priv. comm.). This software package uses the line catalogs from JPL and CDMS (Poynter \& Pickett 1985; Müller et al. 2001). The important source dependent model input parameters are the temperature, the column density, the source size and the line-width. While the latter one is relatively easy to determine from the data, the former three parameters are partly degenerate and various permutations give reasonable fits. Figure 6 shows the observed lower sideband spectra with reasonable model spectra overlayed, the exact model input parameters are stated in each panel. The most important value for the characterization of the 


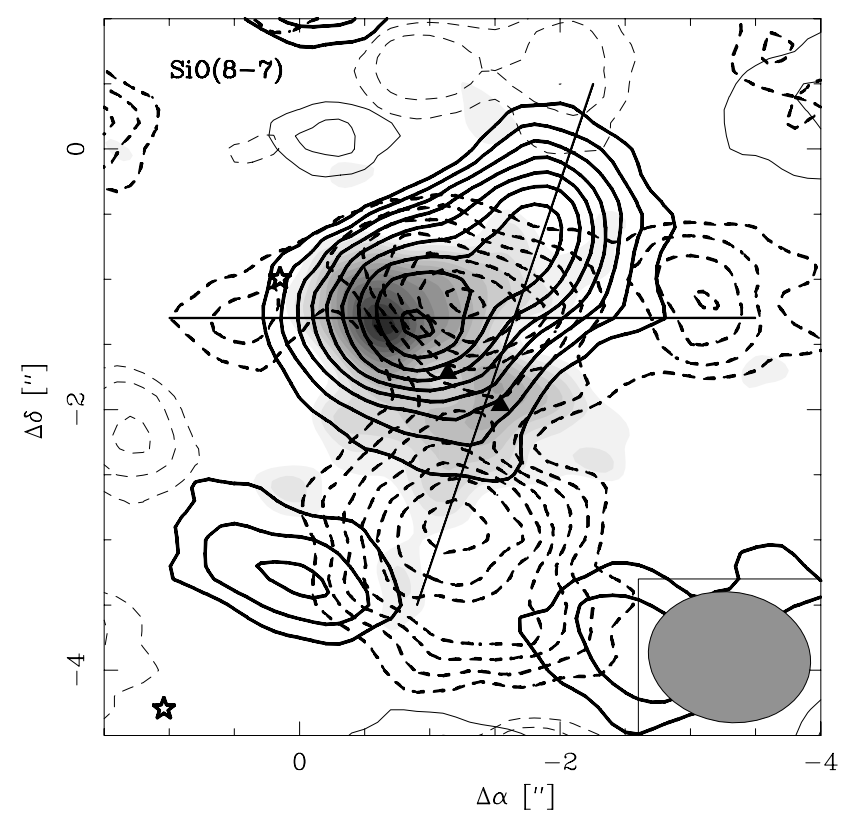

Fig. 4. The full and dashed contours show the blue- and red-shifted $\mathrm{SiO}(8-7)$ emission for IRAS $23151+5912$ integrated from -60 to -56 and from -54 to $-51 \mathrm{~km} \mathrm{~s}^{-1}$, respectively. Thick and thin lines present positive and negative features, the latter ones being due to the incomplete uv-coverage of interferometer datasets. The two lines highlight the two tentatively identified molecular outflows, and the synthesized beam is shown at the bottom-right. The grey-scale shows the submm continuum emission as presented in Fig. 1. Triangles and stars mark the $\mathrm{H}_{2} \mathrm{O}$ maser and near-infrared peaks from Beuther et al. (2002d) and Weigelt et al. (2006). The contour levels go from 18 to $98 \%$ (step 10\%) of the peak emission with blue- and red-shifted peak values of 552 and $445 \mathrm{mJy}$ beam $^{-1}$, respectively.

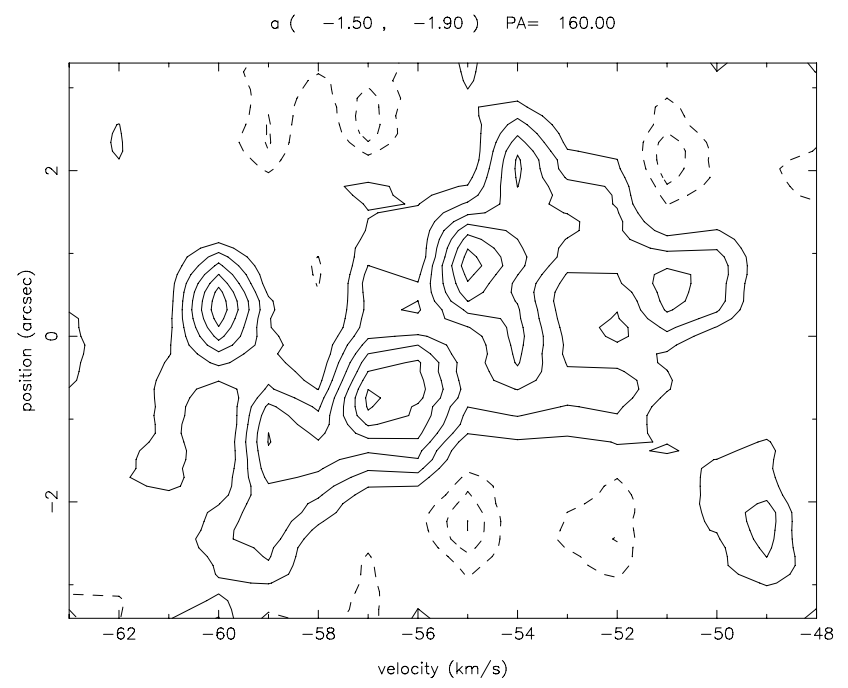

Fig. 5. $\mathrm{SiO}(8-7)$ position-velocity digram of the north-east south-west outflow centered on submm peak 2 (offset $-1.5^{\prime \prime} /-1.9^{\prime \prime}$ from the phase center) with a position angle of 160 degrees from north. Negative offsets are going north-west. The contour levels go from \pm 0.122 to $\pm 0.732 \mathrm{Jy} \mathrm{beam}^{-1}$ in steps of $\pm 0.122 \mathrm{Jy} \mathrm{beam}^{-1}$.

two cores is the temperature. Therefore, we went through the parameter space of mainly temperatures and column density to estimate the uncertainty. The derived temperatures with approximate error-bars for the two positions are $T($ Peak1) $\sim 150 \pm 50 \mathrm{~K}$ and $T($ Peak2) $\sim 80 \pm 30 \mathrm{~K}$. Although the error-bars are large, as expected from the detection of the vibrationally-torsionally
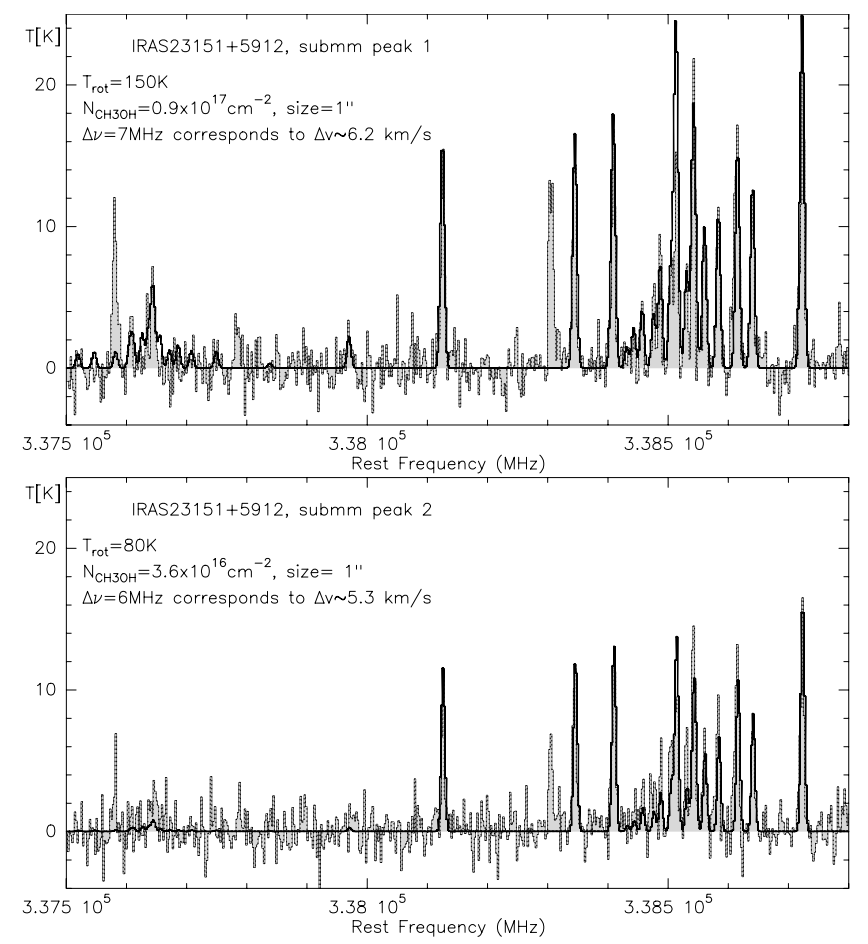

Fig. 6. Lower sideband spectra toward the two submm peaks. The greyscale and dotted contours show the orginal spectrum at $1.1^{\prime \prime} \times 0.9^{\prime \prime}$ resolution, and the thick solid lines present synthetic spectra assuming Local Thermodynamic equilibrium with the parameters stated in the figure.

excited line at the location of submm peak 1 , the data indicate that temperatures toward that peak are likely to be higher.

\subsection{Rotational signatures}

One of the missing links in understanding high-mass star formation is still the detailed understanding of massive accretion disks (e.g., Cesaroni et al. 2007; Beuther et al. 2007a). Therefore, we searched the spectral-line data-cube for rotational signatures perpendicular to any of the molecular outflows. Similar to some other recently investigated regions (e.g., IRAS 18089-1732 Beuther et al. 2005c, G29.96 Beuther et al. 2007b), most of the lines do not show any coherent velocity signature indicative of rotation. The only exception is the vibrationally-torsionally excited methanol line $\mathrm{CH}_{3} \mathrm{OH}\left(7_{1,7}-6_{1,6}\right) v_{\mathrm{t}}=1$ which shows a blue-red velocity shift in north-south direction around submm peak 1 (Fig. 7). Since $\mathrm{CH}_{3} \mathrm{OH} v_{\mathrm{t}}=1$ has an upper level excitation temperature of $\sim 356 \mathrm{~K}$ (Table 3 ) it traces only the warm gas likely excited by the central growing massive protostar. Furthermore, this velocity gradient is perpendicular to the main outflow (Sect. 4.1 and Fig. 4), which likely indicates rotational signatures caused by an underlying collapsing rotational coredisk system (e.g., Cesaroni 2005). The separation between the two blue- and red-shifted $\mathrm{CH}_{3} \mathrm{OH} v_{\mathrm{t}}=1$ peaks is approximately $4300 \mathrm{AU}$. In addition to the relatively large size of the rotating structure, the moment 1 map and position-velocity digram in Fig. 7 do not show a Keplerian velocity pattern as observed for many low-mass sources (e.g., Simon et al. 2000). A Keplerian profile would show the largest velocities close to the center with a velocity decrease proportional to $r^{-1 / 2}$, whereas here we see even a velocity increase with distance from the center. Therefore, this structure is not a Keplerian accretion disk but it rather resembles a larger-scale rotating toroid that may harbor a 

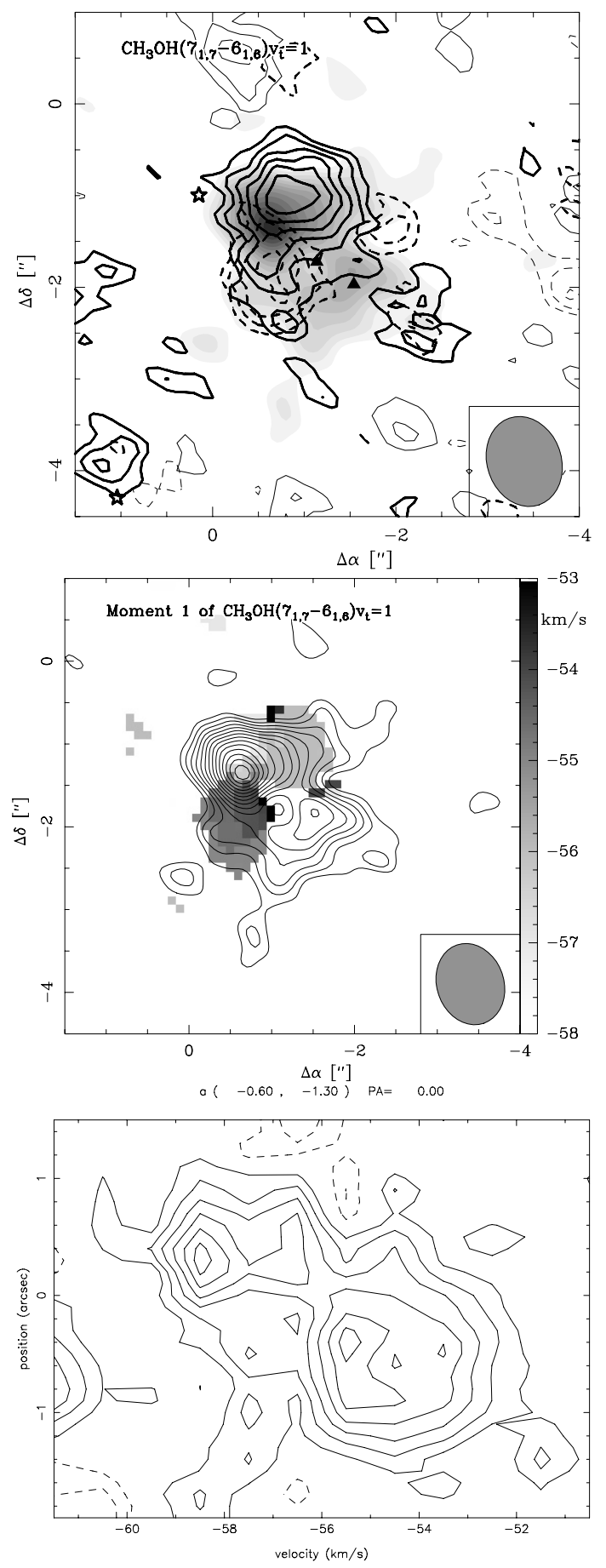

Fig. 7. $\mathrm{CH}_{3} \mathrm{OH}\left(7_{1,7}-6_{1,6}\right) v_{\mathrm{t}}=1$ emission for IRAS 23151+5912. Toppanel: the full/dashed contours show the blue-/red-shifted emission integrated from -58.5 to -55.5 and from -54 to $-52 \mathrm{~km} \mathrm{~s}^{-1}$, respectively. Thick and thin lines present positive and negative features, the latter ones being due to the incomplete uv-coverage of interferometer datasets. The grey-scale shows the submm continuum emission as presented in Fig. 1. Triangles and stars mark the $\mathrm{H}_{2} \mathrm{O}$ maser and nearinfrared peaks (Beuther et al. 2002d; Weigelt et al. 2006). The contour levels start at the $\pm 2 \sigma$ levels and continue in $\pm 1 \sigma$ steps with the $1 \sigma$ levels of 87 and $100 \mathrm{mJy}^{-1}$ beam $^{-1}$ for the blue- and red-shifted maps, respectively. Middle panel: the grey-scale shows the 1st moment map and the contours outline the submm continuum emission as presented in Fig. 1 (The online-version shows this panel in color.). Bottom panel: position-velocity diagram through the submm peak position (offset $-0.6^{\prime \prime} /-1.3^{\prime \prime}$ from the phase reference center) in north-south direction. The contour levels go from \pm 0.136 to $\pm 0.817 \mathrm{Jy}$ beam $^{-1}$ in steps of $\pm 0.136 \mathrm{Jy}^{\text {beam }}{ }^{-1}$. genuine accretion disk at its so far unresolved center (e.g., Cesaroni 2005).

Although this structure is apparently not in Keplerian rotation, one can nevertheless estimate an approximate binding mass of this rotating structure $M_{\text {rot }}$ assuming equilibrium between the rotational and gravitational forces at the outer radius. Then one gets

$$
\begin{aligned}
M_{\text {rot }} & =\frac{v^{2} r}{G} \\
\Rightarrow M_{\text {rot }}\left[M_{\odot}\right] & =1.13 \times 10^{-3} \times v^{2}\left[\mathrm{~km} \mathrm{~s}^{-1}\right] \times r[\mathrm{AU}]
\end{aligned}
$$

where $r$ is the radius of the rotating structure $(\sim 2150 \mathrm{AU}$, half the peak separation in Fig. 7 top panel), and $v$ the Half Width Zero Intensity (HWZI) of the spectral line $\left(\sim 3 \mathrm{~km} \mathrm{~s}^{-1}\right.$, half the velocity regime used for the integration in Fig. 7 top panel). Equations (1) and (2) have to be divided by $\sin ^{2}(i)$ where $i$ is the unknown inclination angle between the plane of the rotating structure and the plane of the sky $\left(i=90^{\circ}\right.$ for an edge-on system). With the given values we get a mass $M_{\text {rot }}$ of $\sim 22 /\left(\sin ^{2}(i)\right) M_{\odot}$, similar to the mass value derived for Peak 1 from the submm continuum emission (Table 2). Assuming an inclination angle of 45 degrees, the rotationally supported mass would be even $44 M_{\odot}$. Although higher than the gas mass derived from the dust continuum emission, this is still within the error margins of the dust continuum calculation which is usually estimated to be correct within factors 2-5 (Beuther et al. $2002 \mathrm{~b}$ ). Assuming that the submm continuum emission largely stems from the disk and/or rotating envelope, this implies that the disk/envelope mass has to be of the same order than the mass of the central embedded protostar. This is different to typical low-mass protostar-disk systems where the disk-mass is usually negligable to the protostellar mass. However, one has to note that such typical low-mass systems are usually more evolved and have already dispersed much of their envelopes whereas IRAS $23151+5912$ is still deeply embedded within its natal core. In addition to this, as shown above, the rotating structure is not in real Kelplerian rotation, and therefore the assumption of equilibrium between gravitational and rotational force does exactly hold from the beginning.

\section{Conclusion and summary}

We present submm line and continuum observations obtained at sub-arcsecond spatial resolution with the SMA toward the very young $10^{5} L_{\odot}$ High-Mass Protostellar Object IRAS 23151+5912. The submm continuum emission is resolved into two sub-sources, each of them likely driving one of the molecular outflows observed in $\mathrm{SiO}(8-7)$. The main submm continuum peak is approximately $1^{\prime \prime}$ offset from a recently identified near-infrared source which is suggested to drive one of the molecular outflows (Weigelt et al. 2006). With these data, we cannot differentiate whether this offset is real indicating the presence of an additional source, or whether it is an observational artifact caused by the relatively poorer near-infrared astrometry. The detection of an intermediate dense spectral line forest with 27 lines within the $4 \mathrm{GHz}$ bandpass allows the investigation of additional physical and chemical properties. The $19 \mathrm{CH}_{3} \mathrm{OH}$ lines provide temperature estimates toward the two main submm continuum peaks to $T($ Peak 1$) \sim 150 \pm 50 \mathrm{~K}$ and $T$ (Peak2) $\sim 80 \pm 30 \mathrm{~K}$, respectively. Searching the dataset for velocity signatures indicative of rotation around the central protostar, only a vibrationally-torsionally excited $\mathrm{CH}_{3} \mathrm{OH}$ line exhibits a velocity gradient perpendicular to the main molecular 
outflow. With a size-scale of a few 1000 AU and no Keplerian velocity profile, we are likely not observing a real accretion disk but rather a larger-scale rotating toroid which may harbor a genuine accretion disk at its very center.

Setting IRAS 23151+5912 into context with lower luminosity HMPOs and higher luminosity UCHIIs, its observed physical and chemical parameters (e.g., line forest, outflow morphology, rotation signatures) are a mixture of what is observed in the two other classes. This supports the idea that this region is in a kind of transition phase between the HMPO and the UCHII region stage. In a follow-up paper, we will present a comparison of the chemical properties of this regions with a few sources representative of different evolutionary stages and luminosities observed all in the same spectral setup with the SMA (Beuther et al. subm. to $\mathrm{A} \& \mathrm{~A})$.

Acknowledgements. We like to thank Peter Schilke for providing the XCLASS software to model the $\mathrm{CH}_{3} \mathrm{OH}$ spectra. Furthermore, we appreciated a lot the discussions with Thomas Preibisch about the infrared emission of this region. In addition, we like to thank the anonymous referee whos comments helped to clarify the paper at several points. H.B. acknowledges financial support by the Emmy-Noether-Program of the Deutsche Forschungsgemeinschaft (DFG, grant BE2578).

\section{References}

Beltrán, M. T., Cesaroni, R., Neri, R., et al. 2004, ApJ, 601, L187 Beltrán, M. T., Brand, J., Cesaroni, R., et al. 2006, A\&A, 447, 221 Beuther, H., Schilke, P., Gueth, F., et al. 2002a, A\&A, 387, 931 Beuther, H., Schilke, P., Menten, K. M., et al. 2002b, ApJ, 566, 945 Beuther, H., Schilke, P., Sridharan, T. K., et al. 2002c, A\&A, 383, 892 Beuther, H., Walsh, A., Schilke, P., et al. 2002d, A\&A, 390, 289 Beuther, H., Schilke, P., Menten, K. M., et al. 2005a, ApJ, 633, 535 Beuther, H., Zhang, Q., Greenhill, L. J., et al. 2005b, ApJ, 632, 355 Beuther, H., Zhang, Q., Sridharan, T. K., \& Chen, Y. 2005c, ApJ, 628, 800 Beuther, H., Churchwell, E. B., McKee, C. F., \& Tan, J. C. 2007a, in Protostars and Planets V, ed. B. Reipurth, D. Jewitt, \& K. Keil, 165

Beuther, H., Zhang, Q., Bergin, E. A., et al. 2007b, A\&A, 468, 1045

Cabrit, S., \& Bertout, C. 1992, A\&A, 261, 274

Cesaroni, R. 2005, Ap\&SS, 295, 5
Cesaroni, R., Churchwell, E., Hofner, P., Walmsley, C. M., \& Kurtz, S. 1994, A\&A, 288, 903

Cesaroni, R., Felli, M., Jenness, T., et al. 1999, A\&A, 345, 949

Cesaroni, R., Galli, D., Lodato, G., Walmsley, C. M., \& Zhang, Q. 2007, in Protostars and Planets V, ed. B. Reipurth, D. Jewitt, \& K. Keil, 197

Claussen, M. J., Marvel, K. B., Wootten, A., \& Wilking, B. A. 1998, ApJ, 507, L79

Eisner, J. A., Hillenbrand, L. A., Carpenter, J. M., \& Wolf, S. 2005, ApJ, 635, 396

Faúndez, S., Bronfman, L., Garay, G., et al. 2004, A\&A, 426, 97

Forster, J. R., \& Caswell, J. L. 1999, A\&AS, 137, 43

Frerking, M. A., Langer, W. D., \& Wilson, R. W. 1982, ApJ, 262, 590

Garay, G., Rodríguez, L. F., \& de Gregorio-Monsalvo, I. 2007, AJ, 134, 906

Hildebrand, R. H. 1983, QJRAS, 24, 267

Hoare, M. G., Kurtz, S. E., Lizano, S., Keto, E., \& Hofner, P. 2007, in Protostars and Planets V, ed. B. Reipurth, D. Jewitt, \& K. Keil, 181

Hunter, T. R., Testi, L., Zhang, Q., \& Sridharan, T. K. 1999, AJ, 118, 477

Keto, E. 2002, ApJ, 568, 754

Keto, E. 2003, ApJ, 599, 1196

Müller, H. S. P., Thorwirth, S., Roth, D. A., \& Winnewisser, G. 2001, A\&A, 370, L49

Molinari, S., Brand, J., Cesaroni, R., \& Palla, F. 1996, A\&A, 308, 573

Poynter, R. L., \& Pickett, H. M. 1985, Appl. Opt., 24, 2235

Qiu, K., Zhang, Q., Beuther, H., \& Yang, J. 2007, ApJ, 654, 361

Richer, J. S., Shepherd, D. S., Cabrit, S., Bachiller, R., \& Churchwell, E. 2000, Protostars and Planets IV, 867

Sault, R. J., Teuben, P. J., \& Wright, M. C. H. 1995, in Astronomical Data Analysis Software and Systems IV, ASP Conf. Ser., 77, 433

Schilke, P., Walmsley, C. M., Pineau des Forets, G., \& Flower, D. R. 1997, A\&A, 321,293

Scoville, N. Z., Carlstrom, J. E., Chandler, C. J., et al. 1993, PASP, 105, 1482

Shepherd, D. S., Watson, A. M., Sargent, A. I., \& Churchwell, E. 1998, ApJ, 507,861

Simon, M., Dutrey, A., \& Guilloteau, S. 2000, ApJ, 545, 1034

Sridharan, T. K., Beuther, H., Schilke, P., Menten, K. M., \& Wyrowski, F. 2002, ApJ, 566, 931

Stark, D. P., Whitney, B. A., Stassun, K., \& Wood, K. 2006, ApJ, 649, 900

Tofani, G., Felli, M., Taylor, G. B., \& Hunter, T. R. 1995, A\&AS, 112, 299

Walmsley, M. 1995, in Revista Mexicana de Astronomia y Astrofisica Conference Series, 137

Weigelt, G., Beuther, H., Hofmann, K.-H., et al. 2006, A\&A, 447, 655

Wilking, B. A., Claussen, M. J., Benson, P. J., et al. 1994, ApJ, 431, L119

Williams, S. J., Fuller, G. A., \& Sridharan, T. K. 2004, A\&A, 417, 115

Wood, D. O. S., \& Churchwell, E. 1989, ApJ, 340, 265

Zhang, Q., Hunter, T. R., \& Sridharan, T. K. 1998, ApJ, 505, L151 